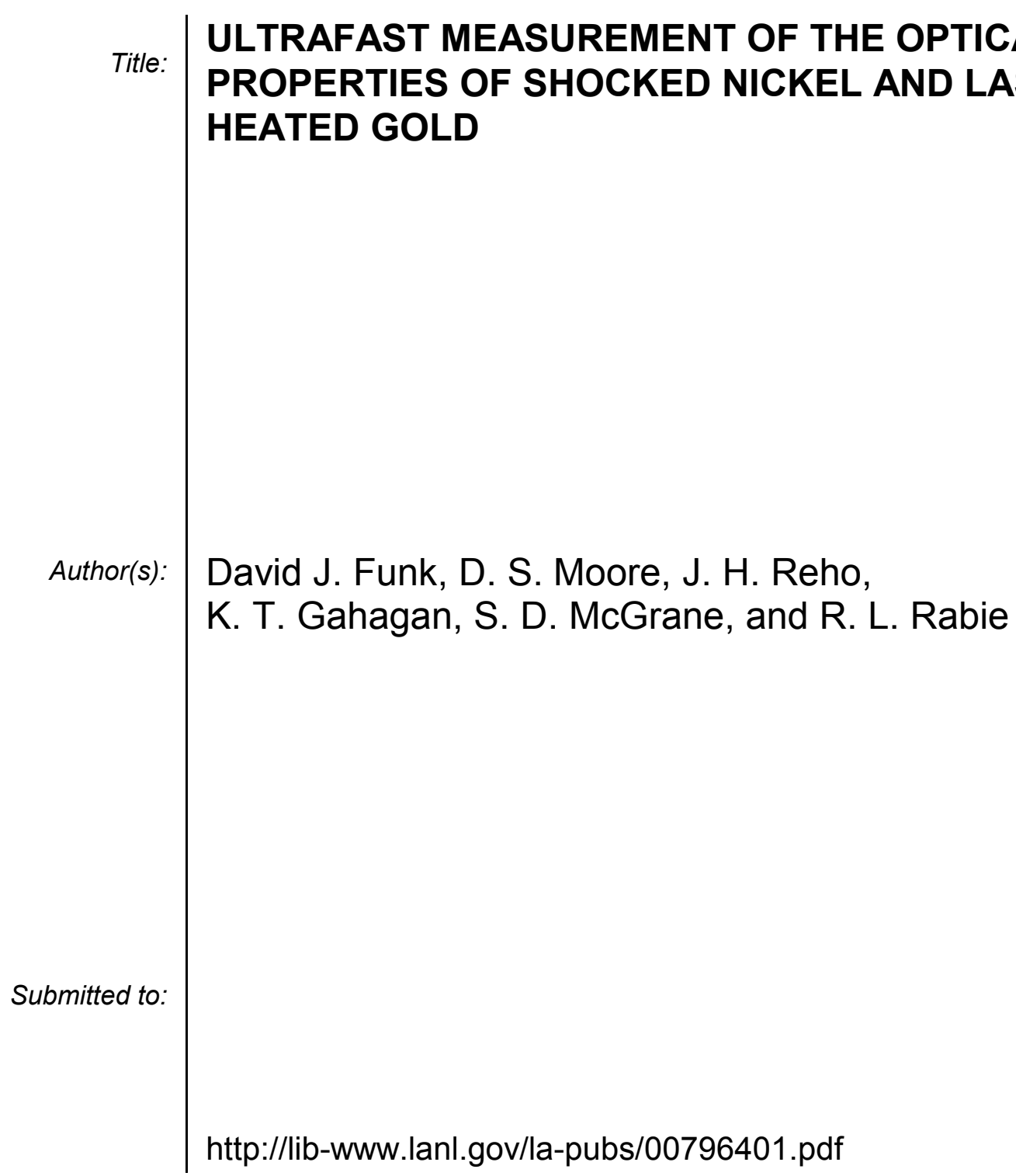

Los Alamos National Laboratory, an affirmative action/equal opportunity employer, is operated by the University of California for the U.S. Department of Energy under contract W-7405-ENG-36. By acceptance of this article, the publisher recognizes that the U.S. Government retains a nonexclusive, royaltyfree license to publish or reproduce the published form of this contribution, or to allow others to do so, for U.S. Government purposes. Los Alamos National Laboratory requests that the publisher identify this article as work performed under the auspices of the U.S. Department of Energy. Los Alamos National Laboratory strongly supports academic freedom and a researcher's right to publish; as an institution, however, the Laboratory does not endorse the viewpoint of a publication or guarantee its technical correctness. 


\title{
ULTRAFAST MEASUREMENT OF THE OPTICAL PROPERTIES OF SHOCKED NICKEL AND LASER HEATED GOLD
}

\author{
David J. Funk, D. S. Moore, J. H. Reho, K. T. Gahagan' ${ }^{1}$, S. D. McGrane, and R. L. \\ Rabie \\ Dynamic Experimentation Division \\ Los Alamos National Laboratory \\ Los Alamos, NM 87545 \\ ${ }^{1}$ Present Address, Corning Incorporated, Corning, NY
}

\begin{abstract}
We have used high-resolution Frequency Domain Interferometry (FDI) to make the first ultrafast measurement of shock-induced changes in the optical properties of thin nickel $(\sim 500 \mathrm{~nm})$ targets. Data taken at several angles of incidence allowed the separation of optical effects from material motion, yielding an effective complex index for the shocked material. In contrast to our previous studies of aluminum, measurements with an $800 \mathrm{~nm}$ probe wavelength found a phase shift attributable to optical property changes with the same sign as that due to surface motion, during an $11.5 \mathrm{GPa}$ shock breakout. A similar experiment was attempted with thin gold films $(\sim 180 \mathrm{~nm})$ using Ultrafast Spatial Interferometry (USI). However, since the electron-phonon coupling in gold is extremely weak, a shock is observed as it "forms". Ballistic electrons and electron-electron equilibrium cause fast heating of the electrons in the entire thickness of the thin film, followed by lattice excitation through electron-phonon coupling, eventually leading to melt and frustrated thermal expansion yielding the observed surface motion. We suggest that these experiments offer a new path for observation of phase changes or for temperature measurements, by allowing a determination of the complex index under dynamic loading conditions and comparing the measured values to those obtained under static conditions.
\end{abstract}

\section{INTRODUCTION}

Development of optical diagnostics that are complementary to $\mathrm{X}$-rays or pyrometric measurements, i.e., diagnostics that are sensitive to phase changes in shocked metallic systems (i.e. melt or solid-solid), or that yield values for the complex index of shocked systems, will help revolutionize the field of shock physics. In addition to the volumetric changes that accompany firstorder phase changes, discontinuities occur in the complex index of refraction (which describes the linear interaction of the material with electromagnetic fields), for both first- and second- order phase changes. Using interferometric techniques, we are able to measure the changes in the complex index during, and for hundreds of ps after, shock wave breakout at free or windowed surfaces. Thus, we suggest that the changes in complex conductivity be used as an indicator of phase or to dynamically measure emissivity.

We have recently used such ultrafast interferometric techniques to characterize shock wave breakout in thin aluminum films [1,2]. The interferometric techniques are capable of resolving sub-nanometer surface deformations with $\sim 100$ femtosecond time resolution and are also sensitive to the dynamic optical properties resulting from shock wave or thermal loading $[1,2]$. We have 
applied the same techniques to studies of shocked nickel and have examined the dynamics of pulse heated gold. In both experiments, changes in the optical properties of the materials are observed and in the case of gold, we attribute the changes to melt, hinting at the possibility of observing shockinduced phase changes with these techniques.

\section{EXPERIMENTAL}

Thin nickel (in-house) and gold (CVI) films of $\sim 500 \mathrm{~nm}$ and $\sim 180 \mathrm{~nm}$ thickness, respectively, were vapor plated onto microscope slide cover slips or white glass. The shock or heating pulses were created in the films by the deposition of laser light from a chirped pulse amplified Ti:sapphire laser. The pulses from this laser are ca. 100-200 fs (FWHM) long at a wavelength of $800 \mathrm{~nm}$ with between $250-500 \mu \mathrm{J}$ of energy per pulse. The illuminated spot diameter is nominally $75 \mu \mathrm{m}$. Two types of interferometry have been used. FDI was used to acquire the nickel data as in $[1,2]$. USI was used to acquire the gold data and the

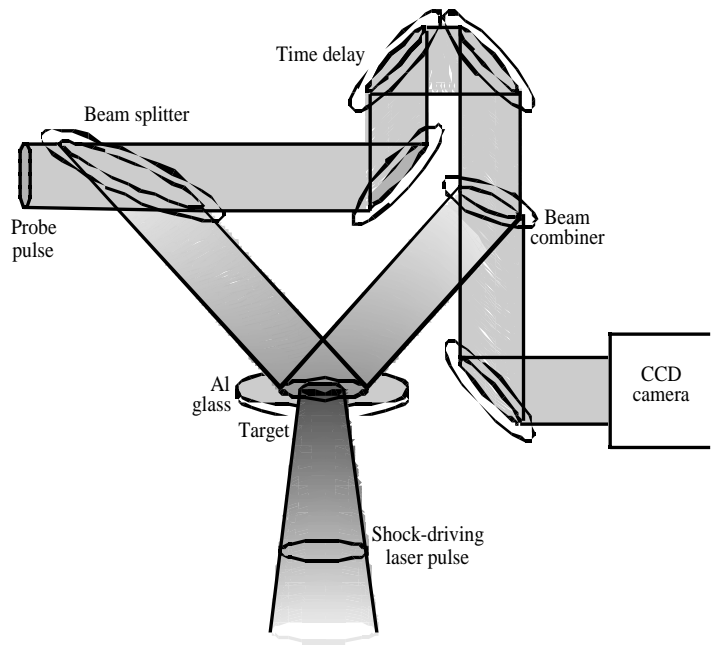

FIGURE 1. Schematic diagram of the femtosecond laser-driven shock/spatial interferometry experiment on thin film aluminum samples.

technique is discussed elsewhere in these proceedings [3]. In both cases, phase and reflectivity data are acquired dynamically.
The experimental arrangement for USI is shown schematically in Fig. 1. The thin film targets were mounted on a computer-controlled $x-y$ translation stage. The target was rastered about 4 laser diameters between experiments so that each laserdriven shock would propagate into undisturbed material. Experiments have been performed in the above sample orientation, with the shock-driving laser focussed through the glass substrate onto the nickel and gold films, as well as in the reverse orientation with gold, with the shock-driving laser focussed onto the gold film side. The probe pulses observed the free gold/nickel surface in the orientation shown in Fig. 1 and the gold/glass interface in the reverse orientation. The time delay between the probe pulses and the the shock-driving laser pulse were adjusted with better than $100 \mathrm{fs}$ precision using computer controlled precision translation stages (Newport Corp.). The two probe pulses interfere either in the exit plane of a spectrometer (FDI) or directly in the focal plane of the CCD (USI). The resulting CCD image for each experiment was analyzed using Fourier transform methods, as in $[2,3,4]$ to extract the difference in phase between the two probe pulses caused by changes in the film surface position or the optical properties of the film. Data were taken at two or three angles to separate out the optical effects from material motion.

\section{RESULTS}

Shown in Fig. 2 are the FDI data and fits obtained for the nickel thin film. In contrast to the FDI results obtained previously for aluminum [1], the data taken near the quasi-polarizing angle (78.3 ) exhibit a positive phase shift attributable to changes in the optical properties, in the same direction as that due to surface motion. Thus, if one naively had taken data only at $32.6^{\circ}$, one could have incorrectly determined the particle velocity and resulting shock state, by not accounting for the contribution due to optical properties. Shown in Figure 3 and 4 are the reflectivity and phase data for the laser-heated gold taken at two angles of incidence and pumping the bare gold (Fig. 3) and through-glass (Fig. 4). It both cases, note the early 
negative phase shift in the $75^{\circ}$ data. Also note that the first changes in phase and/or reflectivity occur near $-42 \mathrm{ps}$. Time zero in these experiments was determined by pumping a standard $250 \mathrm{~nm}$ aluminum film through glass and using "breakout" of the shock as the zero. Given that the shock velocity in aluminum is $\sim 6 \mathrm{~nm} / \mathrm{ps}$, it would take $\sim 42$ ps for the shock to transit the thin film. Thus, the first signals in the gold occur nearly simultaneously with the pump, which would at first appear contrary, but can be explained as resulting from the weak electron-phonon coupling in gold.

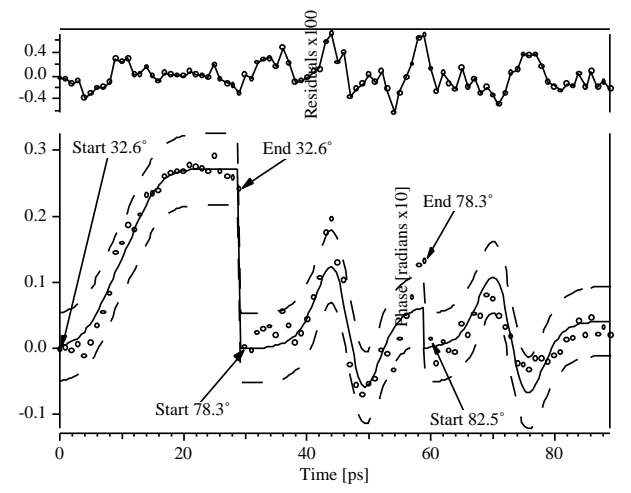

FIGURE 2. Plot of measured phase difference between the probe beams vs. relative delay (to the pump laser) for shock wave breakout from a $500 \mathrm{~nm}$ thick $\mathrm{Ni}$ thin film. The data were obtained using p-polarized $800 \mathrm{~nm}$ light and taken at three angles of incidence (which are offset in time for clarity). Note the large positive phase difference in the data taken near the quasi-polarizing angle in nickel $\left(78.3^{\circ}\right)$, indicating significant complex index changes leading to phase differences of the same sign as those from surface motion. The dashed lines are $95 \%$ confidence limit prediction bands.

\section{DISCUSSION}

To extract a particle velocity, rise time, and the complex index for the shocked nickel data, the data was fit as previously [1], using the following expression for the measured phase shift for each pulse as a function of time:

$\phi_{\mathrm{j}}(t)=\Delta \phi_{n, n_{\text {eff }, k, k_{\text {eaf }}}} \operatorname{sech}^{2}\left(\frac{t-t_{\mathrm{j}}+\delta t_{\mathrm{j}}}{\tau}\right)+\frac{4 \pi \cos \left(\theta_{\mathrm{o}}\right)}{\lambda_{\mathrm{o}}} \int_{4}^{t_{\mathrm{f}}} u_{\mathrm{p}}\left(1+\tanh \left(\frac{t-t_{\mathrm{j}}+\delta t_{\mathrm{j}}}{\tau}\right)\right) d t$

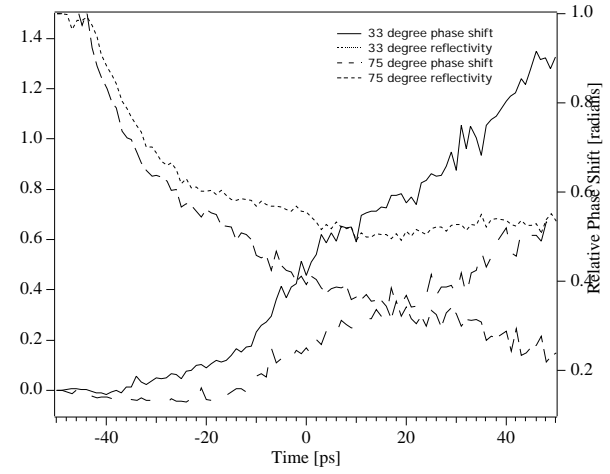

FIGURE 3. Plot of measured phase difference and relative reflectivity between the probe beams vs. delay (relative to the pump laser) at the glass interface of an $180 \mathrm{~nm}$ thick Au thin film. The data were obtained using p-polarized $800 \mathrm{~nm}$ light and taken at two angles of incidence.

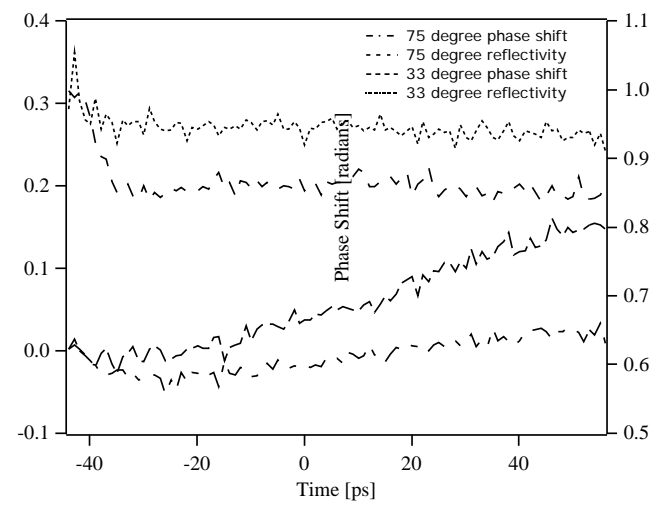

FIGURE 4. Plot of measured phase difference and relative reflectivity between the probe beams vs. delay (relative to the pump laser) at the air interface of an $180 \mathrm{~nm}$ thick Au thin film. The data were obtained using p-polarized $800 \mathrm{~nm}$ light and taken at two angles of incidence.

The functional assumes that the velocity of the free surface has the form of the hyperbolic tangent. It also assumes that the complex index change is a maximum at the peak acceleration, and varies linearly and smoothly from the ambient to the shocked values. The data for the three angles are fit simultaneously by varying the following parameters: $\tau, u_{\mathrm{p}}, t_{\mathrm{j}}, n_{\mathrm{eff}}, k_{\mathrm{eff}}$, where $\tau$ is the hyperbolic tangent time constant, $u_{\mathrm{p}}$ is the shock state particle velocity, $t_{\mathrm{j}}$ are offsets for the data 
from each set. As can be seen in Figure 2, the fits are satisfactory and yield $n=1.56$ and $k=3.35$ for the shocked material's complex index, changing from the ambient values of the nickel $\left(n_{0}=1.6\right.$, $\mathrm{k}_{\mathrm{o}}=3.28$ ) assuming literature values [5]. However, the initial values are likely different from the literature values since Atomic Force Microscope analysis of the films indicate that the RMS roughness of the nickel films is $\sim 7 \mathrm{~nm}$. Finally, the direction of the change in the optical properties is consistent with that of a Drude material under volume compression, which one expects should well represent nickel optical properties, since nickel has no interband transitions near $800 \mathrm{~nm}$.

Concerning the gold data and as noted previously, the first air/Au or glass/Au interface motion occurs nearly simultaneously with the pump laser. Many studies of laser-heated gold have been conducted [6], and have conclusively shown that the electron-phonon coupling in gold is extremely weak, and ca.. 18 times smaller than that in nickel. This results in the following qualitative picture of the laser-material interaction: laser light excites electrons, which, prior to equilibration, have a ballistic penetration depth of $\sim 100 \mathrm{~nm}$ at veocities of $1 \mathrm{~nm} / \mathrm{fs}$. Thus, some fraction of the electrons will penetrate the entire $180 \mathrm{~nm}$ film on a timescale slightly greater than that of the pump laser. The hot electrons will equilibrate with each other through electron-electron collisions, and subsequently with the lattice, through electronphonon coupling. The final temperature of the lattice will depend on the total energy absorbed and the heat capacity of the gold film, with the timescale for equilibrium determined by the electron phonon coupling. The timescales and onset of reflectivity changes, observed in Fig. 2, are consistent with this picture [6].

Finally we would like to make one final observation regarding the data obtained by pumping the bare gold directly. In another study, we believe we have pumped gold to melt using 400 $\mathrm{nm}$ laser light [7]. In that case, the reflectivity at $800 \mathrm{~nm}$ was measured to be approximately $65 \%$ of the original value at an incident angle of 33 degrees. In the experiments we report here, the reflectivity drops to approximately $55 \%$ of the original value at an incident angle of 33 degrees. Though the quantitative extraction of the complex index has not been completed, we believe that, based on the reflectivity measurement and estimates of the gold temperature [8], that, as previously, we have pumped the gold to melt.

\section{CONCLUSIONS}

We have measured changes in the optical properties of shocked nickel and have observed changes in the optical properties of gold while simultaneously measuring the interface position. We believe that these studies pave the way for new investigations of the measurement of temperature and shock-induced phase changes, through correlation of the dynamic complex index with thermodynamic states measured under static conditions.

\section{ACKNOWLEDGEMENT}

This research was performed under the auspices of the US Department of Energy.

\section{REFERENCES}

1. Funk, David J., et al., Phys. Rev. B, in press (2001).

2. Gahagan, K.T., et al., Phys. Rev. Lett. 85(15), 3205-3208 (2000).

3. Gahagan K.T., et al., in this volume p.

4. Evans R., Badger A.D., Failliès F., Mahdieh M., Hall T.A., Audebert P., Geindre J.-P., Gauthier J.-C., Mysyrowicz A., Grillon G., and Antonetti A., Phys. Rev. Lett. 77, 3359 (1996).

5. Smith, D.Y., Shiles, E., and Inokuti, M., in "Handbook of Optical Constants of Solids," E. D. Palik, Ed., p. 374 (Academic Press, San Diego, 1985).

6. Hohlfeld, J., et al., Chem. Phys. 251, 237-258 (2000).

7. Funk David J., unpublished results.

8. Rabie, R. L., unpublished results. The twotemperature model was assumed [7] and a lattice temperature of $\sim 2200 \mathrm{~K}$ obtained, well in excess of melt (1338 K). 